\title{
Projected Potential Profiles across Intergranular Glassy Films
}

\author{
Somnath BHATTACHARYYA, ${ }^{\dagger}$ Christoph T. KOCH and Manfred RÜHLE \\ Max-Planck-Institut fur Metallforschung, HeisenbergStrasse 3, Stuttgart-70569, Germany
}

\begin{abstract}
Projected electrostatic and absorptive potential profiles across intergranular glassy films (IGFs) and interfaces between grains and glassy pockets in silicon nitride ceramics were obtained by reconstructing the electron exit face wave function from a series of defocused TEM images. The phase-object approximation (POA) was used for extracting the projected potential from the complex-valued exit-face wave function. The electrostatic as well as the absorptive potentials were scaled with respect to vacuum. For $\operatorname{La}_{2} \mathrm{O}_{3}-\mathrm{MgO}$ doped $\mathrm{Si}_{3} \mathrm{~N}_{4}$, the potential profiles across the grain/glassy pocket interface and the IGF were observed to be very similar and give very strong evidence for the existence of a space charge layer at the interface.
\end{abstract}

[Received June 2, 2006; Accepted July 27, 2006]

Key-words : Transmission electron microscopy, Exit face wave function, Projected potential profile, Intergranular glassy film, Through focal series, Phase retrieval, Mean inner potential, Space charge

\section{Introduction}

Understanding structure and composition of interfaces is crucial for the design of ceramic materials. In ceramics such as $\mathrm{Si}_{3} \mathrm{~N}_{4}{ }^{1)}$ and $\mathrm{SiC}^{2)}$ 1-2 $\mathrm{nm}$ thick intergranular glassy films (IGFs) are present at the grain boundaries. Although most literature focuses on IGFs in $\mathrm{Si}_{3} \mathrm{~N}_{4}, \mathrm{ZnO}$ and $\mathrm{SiC}$, they have also been observed at grain boundaries in other ceramics (e.g., $\mathrm{SrTiO}_{3},{ }^{3)} \mathrm{Al}_{2} \mathrm{O}_{3}{ }^{4)}$ ), at metal-ceramic interfaces (e.g. $\mathrm{Si}_{3} \mathrm{~N}_{4}$ $\mathrm{Al},{ }^{5)} \quad \mathrm{Al}_{2} \mathrm{O}_{3}-\mathrm{Ni}^{6)}$ ), and at hetero-interfaces in composite creamics (e.g. ruthenate-silicate glass, ${ }^{7)} \mathrm{Si}_{3} \mathrm{~N}_{4}-\mathrm{SiC}^{8)}$ ) etc. Varying the composition of IGFs has profound influence on material properties such as creep resistance, oxidation resistance, fracture toughness etc. Many transmission electron microscopic techniques have been used so far to investigate structure and chemistry of IGFs. The film width has commonly been measured by high resolution lattice fringe imaging, diffuse dark field imaging or Fresnel fringe imaging. ${ }^{3,9)}$ Energy dispersive X-ray spectroscopy (EDXS) and electron energy loss spectroscopy (EELS) have been used to study their chemistry. ${ }^{10), 11)}$ Recently segregation of heavy elements at IGFs was imaged using high-angle annular dark field scanning transmission microscopy (HAADF-STEM). ${ }^{12)-14)}$ High frequency components of the phase across the IGF of yttria doped $\mathrm{Si}_{3} \mathrm{~N}_{4}$ were mapped with atomic resolution using high resolution images at different foci. ${ }^{15)}$ Off-axis electron holography has been used to determine the local electrostatic potential and associated space charge across an IGF $^{16)}$ while electron diffraction experiments were able to provide IGF potential profiles averaged over large distances along the boundary. ${ }^{17)}$

The mean inner potential profile across grain boundaries provides useful information about variations in composition and density across it, as well as about intrinsic potentials associated with depletion layers or space charge. ${ }^{18)}$ The projected electrostatic potential within a TEM specimen induces a phase-shift in the wave function describing a fast electron passing through the specimen, and may therefore be measured by either off-axis or in-line holographic methods. ${ }^{19)}$ Stobbs and coworkers developed an approach to use the Fresnel contrast (contrast in largely defocused images produced by a wave function containing local phase differences, i.e. in-line

\footnotetext{
$\uparrow$ Present address: IFF, Forschungzentrum Jülich, 52425, Jülich, Germany

E-mail: somnath-tem@yahoo.com
}

holography) to analyze potential profiles across interfaces by fitting a model potential profile described by only a few parameter to the fringe contrast. This approach was reviewed and extended further by Dunin-Borkoski. Since variations in projected potential are only expected across, but not along an interface the Fresnel contrast method, in this case, only requires line profiles extracted from a series of images with varying defocus normal to the plane of the defect. Minimizing the difference between experimental and simulated images, Dunin-Borkoswki fitted the interface width, the magnitude of its potential well or hill and the diffuseness of its boundaries to the experimental image intensity profiles by comparing them with simulated ones at every defocus for the entire experimental defocus series. The solution to this global nonlinear optimization problem is a projected potential profile that can be described by the given parameter set and produces Fresnel contrast images most similar to the experimental ones.

Recently, Bhattacharyya et al. ${ }^{20)}$ reported the development of a phase retrieval method suitable for application to energyfiltered Fresnel contrast focal series based on the iterative wave function retrieval (IWFR) algorithm developed by Allen et al. ${ }^{21)}$ This method solves the above mentioned global optimization problem without the need for reducing the parameter space, or limitations on the defocus step size as is the case for the transport-of-intensity-equation (TIE) based approach. ${ }^{22)}$,23) It therefore does not require the assumption of any model shape describing the potential profile. The projected potential may be extracted from the reconstructed wave function using the phase-object approximation (POA).

$$
\psi(\boldsymbol{r})=\exp (\boldsymbol{i} \sigma t V(\boldsymbol{r})),
$$

where $V(r)$ is the projected potential and may be complex. $\Psi(r), \sigma$ and $t$ are the exit face wave function, relativistic electron interaction constant and specimen thickness along the electron beam respectively. The real (electrostatic) part of the projected potential produces a pure phase shift in the exit face wave function, while its imaginary part (responsible for inelastic and high-angle scattering outside the objective aperture, as produced by the presence of atomic species of higher $Z)$ affects only its amplitude.

In this paper, we used the above mentioned method to retrieve potential profiles across IGFs in $\mathrm{Si}_{3} \mathrm{~N}_{4}$, a ceramic material with many industrial applications, whose mechanical properties vary strongly with composition of the IGFs. ${ }^{24)}$ Koch et al. ${ }^{17)}$ reconstructed the shape of the projected poten- 
tial profile across an IGF of $\mathrm{Lu}_{2} \mathrm{O}_{3}-\mathrm{MgO}$ doped $\mathrm{Si}_{3} \mathrm{~N}_{4}$ ceramics with an arbitrary scale from a tilt series of energyfiltered electron diffraction patterns. To the best of our knowledge, this paper contains the first report of the potential profiles across an IGF in a $\mathrm{Si}_{3} \mathrm{~N}_{4}$ ceramic with absolute scale. A new measure of the IGF width, referred to as "potential well width", is also introduced here. For one of the samples, not only the projected potential profiles across the IGF but also across the adjacent grain/glassy pocket interface were determined which gives a new insight about the nature of the potential profiles across the IGF.

\section{Experimental details}

Dense silicon nitride samples with $\mathrm{Lu}_{2} \mathrm{O}_{3}$ and $\mathrm{MgO}$ doping have been prepared by Satet and Hoffmann from milled and sieved $\alpha-\mathrm{Si}_{3} \mathrm{~N}_{4}$ (88.07 wt \%), $\mathrm{SiO}_{2}$ (2.16 wt \%), $\mathrm{MgO}(1.07$ wt $\%)$ and $\mathrm{Lu}_{2} \mathrm{O}_{3}(8.7 \mathrm{wt} \%)$ powders, by first cold pressing, then sintering at $1750^{\circ} \mathrm{C}$ for $15 \mathrm{~min}$ at a pressure of $10 \mathrm{bar}$ and finally hot isostatic pressing (HIP) at $1750^{\circ} \mathrm{C}$ for $60 \mathrm{~min}$ at 100 bar. To prepare the $\mathrm{La}_{2} \mathrm{O}_{3}$ and $\mathrm{MgO}$ doped $\mathrm{Si}_{3} \mathrm{~N}_{4}$ sample, a mixture of milled and sieved $\alpha-\mathrm{Si}_{3} \mathrm{~N}_{4}(88.6 \mathrm{wt} \%)$ with $\mathrm{SiO}_{2}$ (2.20 wt $\%), \mathrm{MgO}(2 \mathrm{wt} \%)$ and $\mathrm{La}_{2} \mathrm{O}_{3}(7.2 \mathrm{wt} \%)$ powders was cold pressed followed by sintering at $1780^{\circ} \mathrm{C}$ for $14 \mathrm{~min}$ at a pressure of $10 \mathrm{bar}$ and finally hot isostatic pressing at $1780^{\circ} \mathrm{C}$ for $30 \mathrm{~min}$ at $100 \mathrm{bar}$.

The specimens for transmission electron microscopy were prepared by the standard techniques of grinding, dimpling and ion-beam thinning (Precision Ion Polishing System, Gatan Inc.). The ion-beam thinning was carried out at an angle of inclination of $8^{\circ}$ of the ion beam to the sample. The $\mathrm{Si}_{3} \mathrm{~N}_{4}$ specimens were coated with a thin layer of carbon to minimize charging under the electron beam.

The experiments were performed using a Zeiss 912 microscope ( $C_{\mathrm{s}}=2.7 \mathrm{~mm}, C_{\mathrm{c}}=2.7 \mathrm{~mm}$, Köhler illumination), operated at $120 \mathrm{kV}$ voltage and equipped with an in-column Omega-type energy filter. Same experimental conditions were maintained for every experiment by using an electron beam illumination semi-angle of $0.25 \mathrm{mrad}$ and an objective aperture of size $0.1248 \AA^{-1}$.

Care was taken to orient the interfaces of interest (e.g. grain boundaries) parallel to the incident electron beam (edge-on condition). The specimens were further tilted, keeping the grain boundaries of interest edge-on, in a way to orient the grains on either side of the boundary to weak diffracting conditions. ${ }^{25)}$

To acquire the energy filtered images, a $15 \mathrm{eV}$ energy slit centered on the zero loss peak was used. All the experimental images were captured onto a $1024 \times 1024$ pixel CCD array using 5 second exposure time at a magnification producing an image scale of about $0.19 \mathrm{~nm}$ per pixel. The through focal series of images were taken from the defocus value of $1 \mu \mathrm{m}$ to $4 \mu \mathrm{m}$ with a step size of $0.5 \mu \mathrm{m}$ in both over and under focus directions. Digital micrograph software (Gatan Inc., Pleasanton, CA, USA) was used to extract line profiles across the same area of the interface from each of the images (line scans were integrated over a width of 100 pixels). The data were processed using programs written in MATLAB 7.0 (The Mathworks Inc., Natick, MA, USA) software to reconstruct the exit face wave function.

\section{Retrieval of the projected potential}

The projected potential was retrieved from the Fresnel contrast image focal series as described by Bhattacharyya et al.. This algorithm reconstructs the exit face way function in an iterative way. The distinct features of this algorithm are:
1. Image drift compensation for images recorded with largely different focus and thus contrast.

2. Numerical stability even in the presence of missing spatial frequency components in the microscope transfer functions at some defoci.

3. Low spatial frequency components of the electrostatic potential may be retrieved by applying prior knowledge of areas of constant potential (e.g. inside the grains, or regions of vacuum).

An outline of the projected potential retrieval procedure is presented in Fig. 1. Finally, the projected potential is split up as

$$
V(r)=V^{\prime}(r)+i V_{\mathrm{abs}}(r),
$$

where, potential $V^{\prime}(r)$ is due to the elastic interaction of the electron with the specimen referred to as the "electrostatic potential', and $V_{\text {abs }}(r)=\left[V^{\prime \prime}(r)+V^{\prime \prime \prime}(r)\right]$ describes the loss of electrons due to inelastic scattering events $\left(V^{\prime \prime}(\boldsymbol{r})\right)$ and elastic scattering outside the objective aperture $\left(V^{\prime \prime \prime}(r)\right)$ and may be called objective aperture dependent 'pseudo-absorptive' scattering potential. ${ }^{20), 26)}$ While the use of a small objective aperture greatly enhances Fresnel contrast it also allows this 'pseudo-absorptive' potential to be mapped with better signal/noise ratio.

In the work reported previously, the reconstructed exit face wave function was normalized with respect to its maximum value (for extracting the projected potential) to eliminate physically insensible negative values in the absorptive potential, i.e. the apparent production of electrons by the sample itself. The scale of the absorptive potential is therefore relative to the least absorbing point within the field of view. A more rigorous normalization is proposed also in that paper where images of all focal planes are divided by a flat field image (an image recorded without a specimen in the field of view) recorded under the same imaging conditions to give the absorptive potential profile an absolute scale. This latter method of normalization has been applied in the work presented here.

Despite its potential the method as implemented for this work also suffers from the limited resolution of $8 \AA$ imposed by the objective aperture. The use of a thermionic $\mathrm{LaB}_{6}$ electron gun also limits the spatial coherence which puts an upper limit on the IGF widths that can be observed. For the present study the film widths have been well within the limits imposed by the spatial coherence. Since the work reported previously showed that dynamic scattering has negligible effect on the reconstructed electrostatic potential, the phase-object approximation can safely be used for the present study.

\section{Results}

The specimen thickness along the direction of the electron beam was determined using only two images, a zero-loss filtered one $\left(I_{0}\right)$ and an unfiltered one $\left(I_{1}\right)$, both recorded with the same exposure time. Using the $1 \mathrm{D}$ intensity profiles taken from the same area of the images as the ones in the focal series reconstruction, the local specimen thickness, $t$, is calculated according to Egerton: ${ }^{27)}$

$$
t=\lambda_{\text {inel }} \ln \left(\frac{I_{1}}{I_{0}}\right)
$$

where $\lambda_{\text {inel }}$ is the inelastic mean free path (IMFP) of the electron in the given material that was calculated as described by Egerton. For $\mathrm{Si}_{3} \mathrm{~N}_{4} \lambda_{\text {inel }}=132.2 \mathrm{~nm}$.

Where no vacuum reference phase was available within the field of view the reconstructed electrostatic potential profile of 


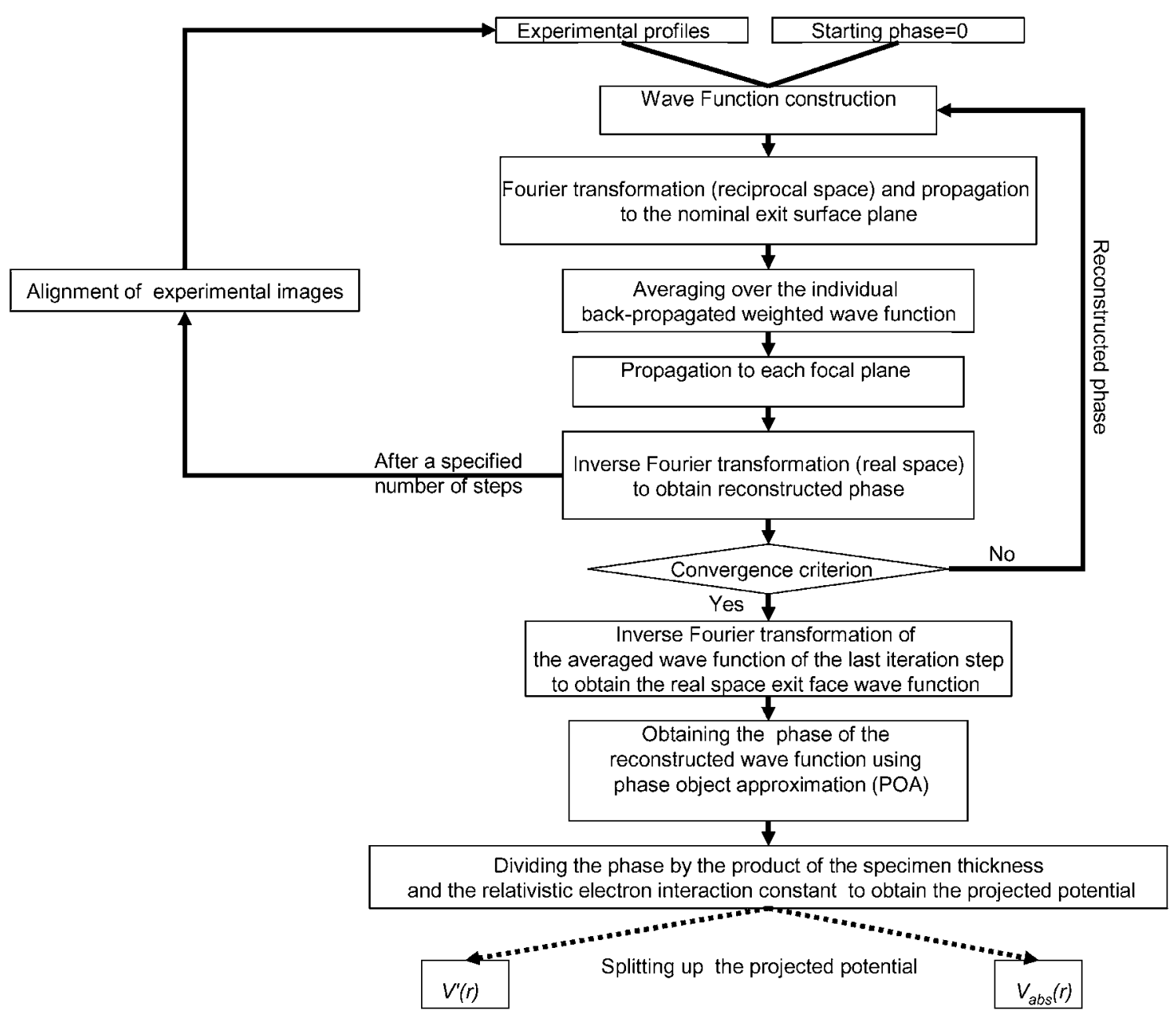

Fig. 1. Outline of the projected potential retrieval procedure.

IGF and $\mathrm{Si}_{3} \mathrm{~N}_{4}$ grain/triple pocket interface has been scaled with respect to the mean inner potential of the $\mathrm{Si}_{3} \mathrm{~N}_{4}$ grains experimentally determined from a $\mathrm{Si}_{3} \mathrm{~N}_{4}$ /vacuum interface. This is possible because of the insensitivity of the specimen induced electron wave phase shift on grain orientation, i.e. the local diffraction condition. Just as in off-axis holography, ${ }^{28)}$ the estimates for the electrostatic potential are absolute, since they are made in reference to vacuum, where we know that $V^{\prime}(r)=0$.

\subsection{Vacuum $/ \mathrm{Si}_{3} \mathrm{~N}_{4}$ interface}

An image of the vacuum $/ \mathrm{Si}_{3} \mathrm{~N}_{4}$ interface at an overfocus of $1.1 \mu \mathrm{m}$ is shown in Fig. 2(a). The dotted box shows the area from where the integrated line scan was taken. Some of the aligned and normalized 1D experimental intensity profiles (of different defocus) across the interface are shown in Fig. 2(b). To make the thickness variation smooth, starting from the edge to the sample, the thickness profile was replaced by a fitted 2nd order polynomial as shown in Fig. 2(c). Reconstructed electrostatic and absorptive potentials of this interface are shown in Figs. 2(d) and 2(e) respectively. The vanishing specimen thickness in the vacuum region forced us to set $V^{\prime}(r)=V_{\text {abs }}(r)=0$ for $r<0$.

The absorptive potential consists of the electron scattering outside the objective aperture used in these experiments and the inelastic scattering outside the energy slit of $15 \mathrm{eV}$ width centered at the zero loss peak. The loss of electrons in both, the diffraction plane (by the objective aperture) and the ener- gy-dispersive plane (by the energy slit) produces a positive absorptive potential inside the sample relative to vacuum (Fig. 2(e)).

All experiments were done using partially coherent electron beam illumination. The coherence width $\left(X_{\mathrm{c}}\right)^{29)}$ is the distance at the object over which the illuminating radiation may be treated as perfectly coherent. The coherence width can be calculated as $X_{\mathrm{c}}=\lambda / 2 \pi \theta_{\mathrm{c}}$, where $\lambda$ is the electron wavelength, and $\theta_{c}$ is the illumination semi-angle (the beam divergence). The illumination semi-angle was kept at $0.25 \mathrm{mrad}$ for all the experiments presented here, resulting in a width of $21.3 \AA$ over which the electrons can be treated as being perfectly coherent. For partially coherence electron irradiation, another criterion is the incoherence width $\left(X_{i}=\lambda / \theta_{\mathrm{c}}=134 \AA\right)$, which defines the distance at the object above which the illumination is completely incoherent. Spatial frequency components of the illuminating electron wave function corresponding to wave numbers between $21.3 \AA$ and $134 \AA$ are partially coherent. The present experimental conditions are therefore sufficient for the reconstruction of $V^{\prime}(r)$ across distances relevant to IGFs in $\mathrm{Si}_{3} \mathrm{~N}_{4}$.

Figure $2(\mathrm{~d})$ shows that the change of $V^{\prime}(r)$ from vacuum to $\mathrm{Si}_{3} \mathrm{~N}_{4}$ is not abrupt but gradual. This could be due to the formation of an amorphous layer just near the hole produced during ion milling, the final TEM specimen preparation step. A less dense amorphous material consisting of the same atomic species is expected to have lower amplitude of elec- 


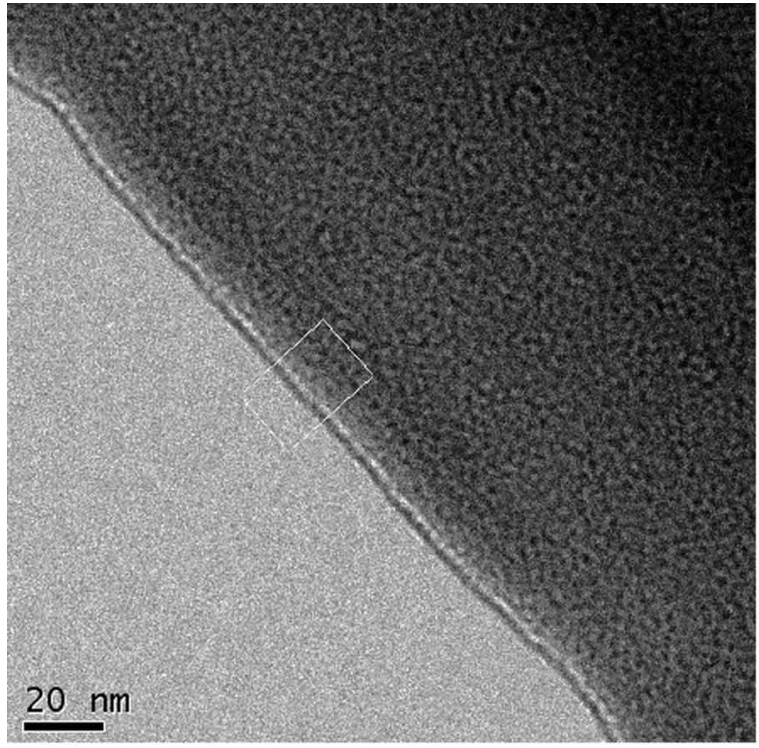

(a)

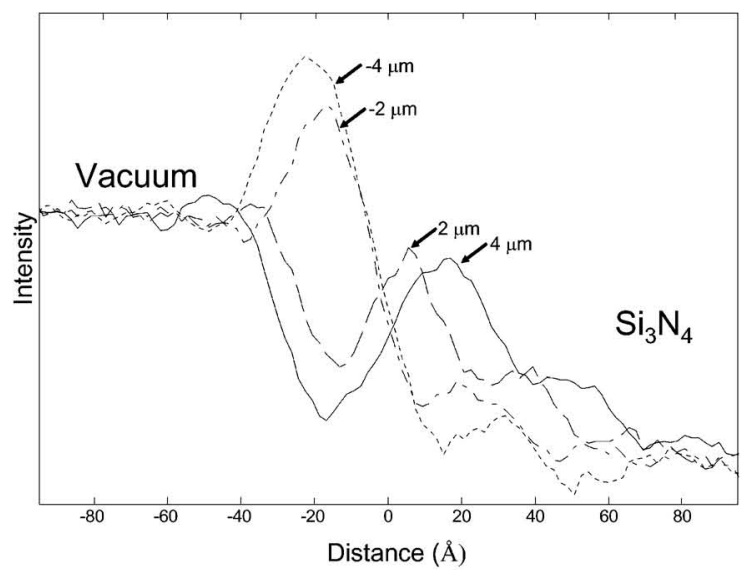

(b)

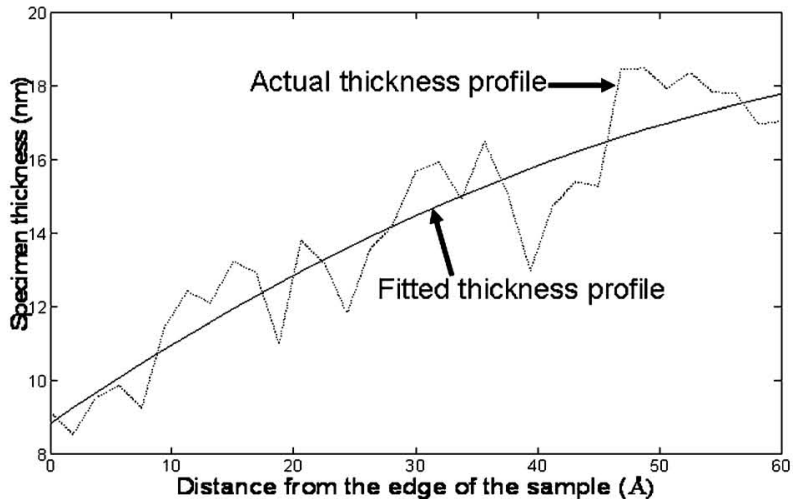

(c)

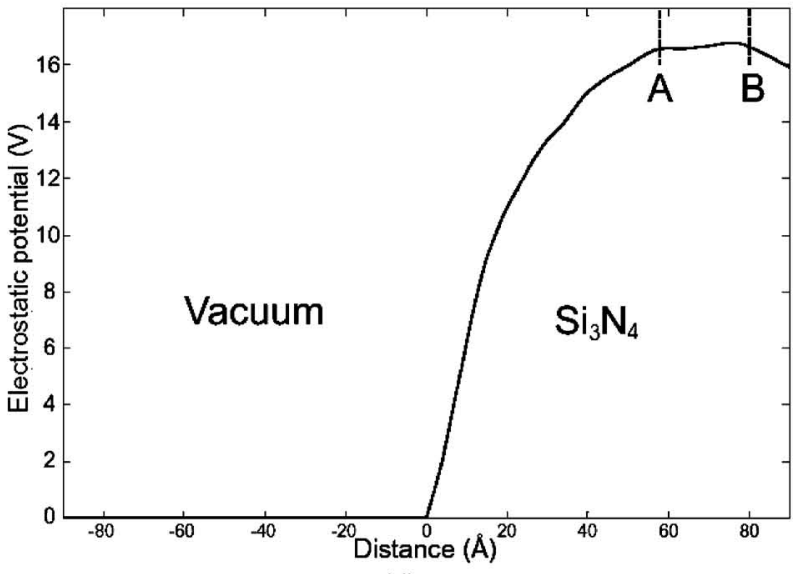

(d)

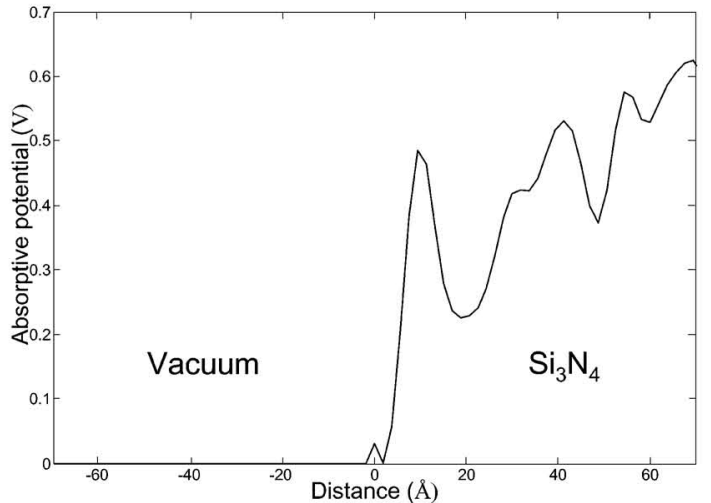

(e)

Fig. 2. (a) Image of a vacuum $/ \mathrm{Si}_{3} \mathrm{~N}_{4}$ interface at an overfocus value of $1.1 \mu \mathrm{m}$. (b) Line profiles across the grain boundary extracted from 4 images of different foci (corresponding defocus values are indicated in the figure usings arrows), (c) specimen thickness along the electron beam (d) reconstructed electrostatic potential (low spatial frequency components were determined by assuming that the vacuum region for - $112.6 \AA$ $<x<-28.9 \AA$ is featureless) and (e) reconstructed absorptive potential.

trostatic potential than its crystalline counter part $\mathrm{Si}_{3} \mathrm{~N}_{4}$.

The average electrostatic potential of $\mathrm{Si}_{3} \mathrm{~N}_{4}$ as determined from the region $\mathrm{AB}$ of Fig. $2(\mathrm{~d})$ is $16.7 \mathrm{~V}$. This experimentally determined mean inner potential of $\mathrm{Si}_{3} \mathrm{~N}_{4}$ is in good agreement with the calculated value $(17.4 \mathrm{~V})$. The discrepancy between the calculated and reconstructed mean inner potential may be due to a slightly wrong estimate of the IMFP of $\mathrm{Si}_{3} \mathrm{~N}_{4}$ (essential for accurate specimen thickness determination) or the neutral atom assumptions on which the calculation of the mean inner potential is based. Beyond point B (Fig. 2(d)) $V^{\prime}(r)$ again decreases due to the limited spatial coherence in the microscope used for this investigation.

\section{$4.2 \mathrm{La}_{2} \mathrm{O}_{3}-\mathrm{MgO}$ doped $\mathrm{Si}_{3} \mathrm{~N}_{4}$}

\subsubsection{Intergranular glassy film}

An image of the grain boundary of interest together with the glassy pocket, at an overfocus value of $3 \mu \mathrm{m}$, is shown in Fig. 3 (a). Here also the dotted boxes show the areas from where the integrated line scans were taken. The specimen thickness along the electron beam direction is presented in Fig. 3(b). As the exact composition of the IGF is not known, the IMFP of $\mathrm{Si}_{3} \mathrm{~N}_{4}$ was also used for the IGF. Since the thickness did not vary much within each of the 2 grains, averaged numbers have been used instead of the more noisy experimentally determined thickness profile (numbers given in Fig. 3 


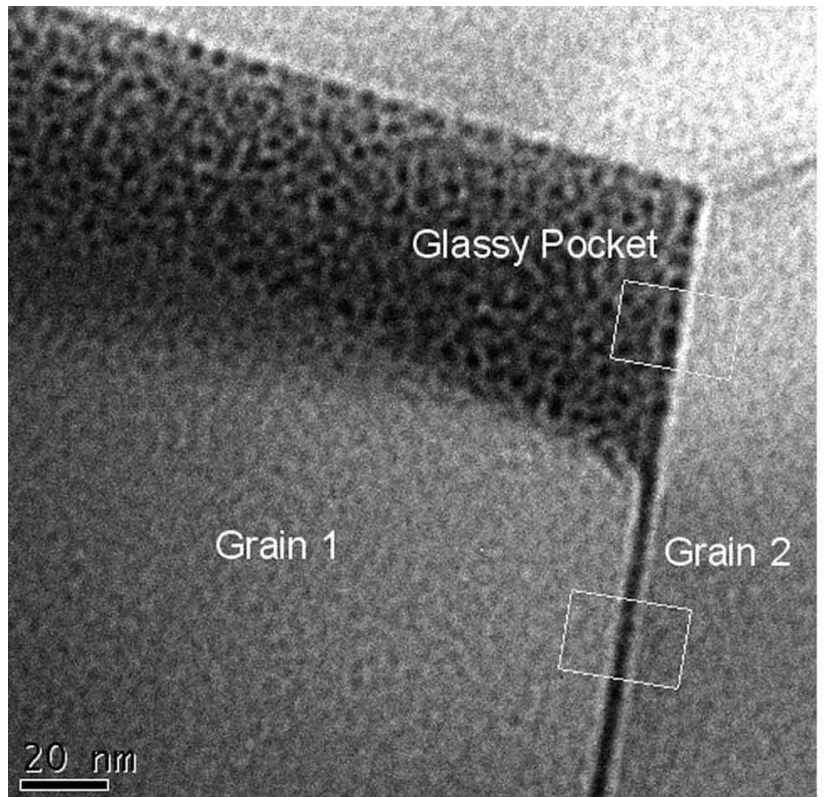

(a)

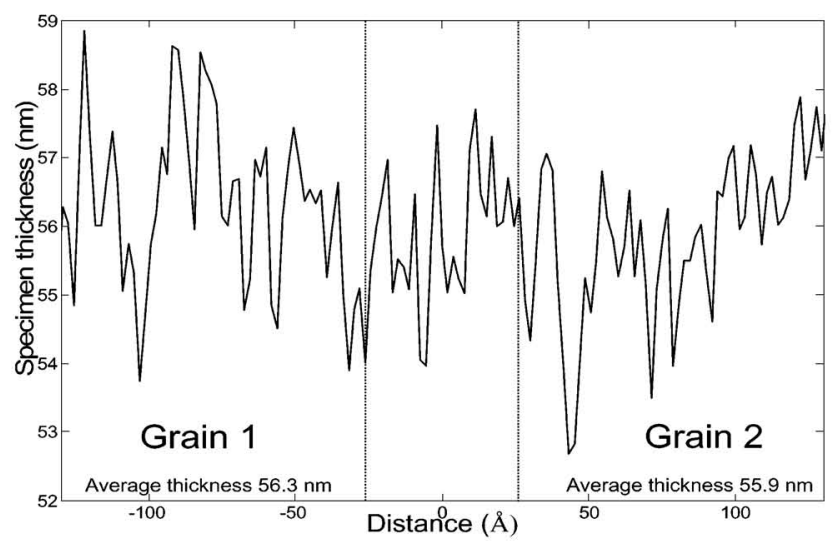

(b)

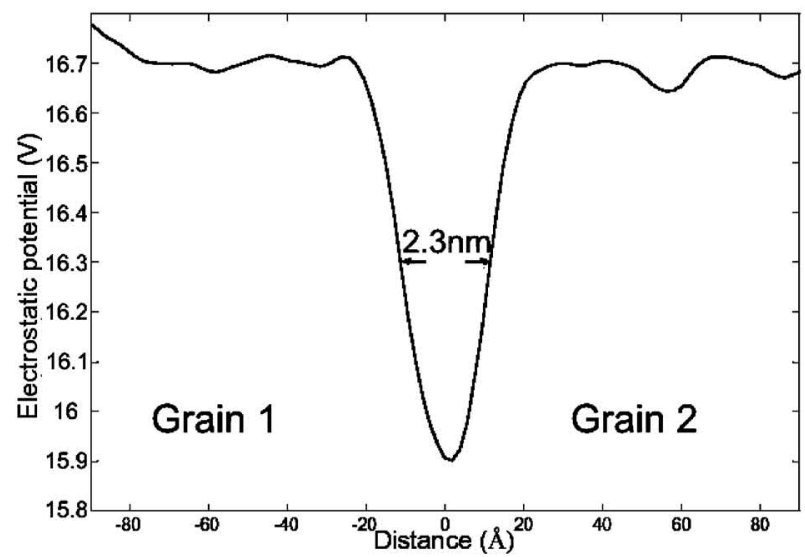

(c)

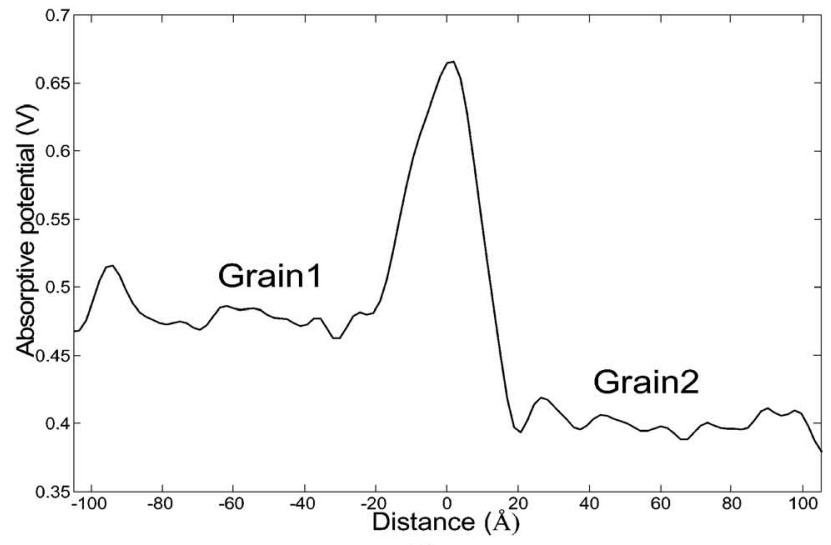

(d)

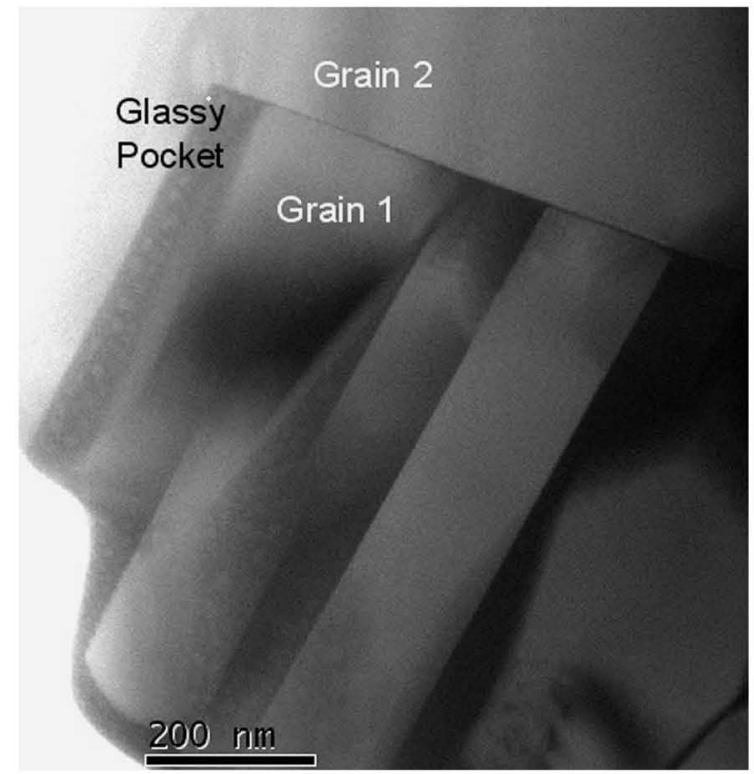

(e)

Fig. 3. (a) Image of an IGF and glassy pocket in a $\mathrm{La}_{2} \mathrm{O}_{3}-\mathrm{MgO}$ doped $\mathrm{Si}_{3} \mathrm{~N}_{4}$ ceramics at an overfocus value of $3 \mu \mathrm{m}$. (b) Specimen thickness along the electron beam (c) reconstructed electrostatic potential (The potential was assumed to be constant for $-101.5 \AA<x<-23.3 \AA$ and also for $40.9 \AA<x<127.6 \AA$ ) and (d) reconstructed absorptive potential across IGF (e) overview of the area at low magnification.

(b)). The mean of these two thickness values was assumed as the specimen thickness across the IGF. This assumption ignores any preferential thinning near the grain boundary due to ion milling.

The scaled (absolute) reconstructed electrostatic and the absorptive potentials across the IGF are presented in Fig. 3 (c) and Fig. 3(d) respectively. The full width at half maximum (FWHM) of the electrostatic potential profile across the IGF will be regarded as the "potential well width" from here on. This width, for the profile shown Fig. 3 (c), is $2.3 \mathrm{~nm}$. This 
figure shows that the IGF has a lower electrostatic potential i.e. less mean inner potential than the $\mathrm{Si}_{3} \mathrm{~N}_{4}$ grains adjacent to it.

Due to different diffracting conditions, the grains on either side of the IGF show different absorptive potentials, as shown in Fig. 3(d). Elastic scattering to large angles may be approximated rather well by the Rutherford elastic scattering cross section which is proportional to the square of the atomic number of the scattering element. As reported by different researchers ${ }^{12)-14)}$ using HAADF-STEM, in $\mathrm{La}_{2} \mathrm{O}_{3}-\mathrm{MgO}$ doped $\mathrm{Si}_{3} \mathrm{~N}_{4}$ ceramics, La atoms segregate at the IGF/grain interface when the prism plane of $\beta-\mathrm{Si}_{3} \mathrm{~N}_{4}$ constitutes the interface plane. In Fig. 3(e), the overview of the examined area at lower magnification, shows that in grain 2 which is a grain with high aspect ratio, the longest plane is the interface plane (IGF/grain interface). According to Petzow and Herrmann, ${ }^{30)}$ in $\beta-\mathrm{Si}_{3} \mathrm{NB}_{4}$ ceramics, the longest planes of the grains with high aspect ratio, are the prism planes. So, here the interface plane of grain 2 is probably the prism plane on which $\mathrm{La}$ atoms segregate. Since the atomic number of $\mathrm{La}$ is $\mathrm{Z}_{\mathrm{La}}=57$ $\left(Z_{S i}=14, Z_{N}=8\right)$, high-angle scattering outside the objective aperture is expected to be larger within the IGF than in the adjacent $\mathrm{Si}_{3} \mathrm{~N}_{4}$ grains. That is why the IGF shows a larger absorptive potential than the grains next to it.

\subsubsection{Grain/glassy pocket interface}

The image of this interface at an overfocus of $3 \mu \mathrm{m}$ is shown in Fig. 3(a). Since the exact composition of the amorphous material in the glassy pocket is not known, it is assumed that the thickness along the beam direction remains constant across the interface and the average thickness of the $\mathrm{Si}_{3} \mathrm{~N}_{4}$ grain at one side of the interface was used for the whole line profile. This assumption ignores preferential thinning in the glassy pocket region during ion milling. The scaled (absolute) electrostatic and the absorptive potentials across this interface are shown in Figs. $\mathbf{4}(\mathrm{a})$ and $\mathbf{4}(\mathrm{b})$ respectively. Assuming the electrostatic potential of the $\mathrm{Si}_{3} \mathrm{~N}_{4}$ grain as the base, the potential well width of this interface is $2.6 \mathrm{~nm}$.

According to Winkelman et al, in this material, La atoms segregate to the glassy pocket/grain interface when prism planes of $\beta-\mathrm{Si}_{3} \mathrm{~N}_{4}$ constitutes the interface plane. So, for the same reason as IGF, the glassy pocket shows a higher absorptive potential than the adjacent grain.

\section{3 $\mathrm{Lu}_{2} \mathrm{O}_{3}-\mathrm{MgO}$ doped $\mathrm{Si}_{3} \mathrm{~N}_{4}$}

An image of the IGF of interest at an overfocus of $2 \mu \mathrm{m}$ is shown in Fig. 5(a). Here also the dotted box indicates the region from which the line profile has been extracted. To determine the specimen thickness along the electron beam we used the same procedure as used for the IGF in the $\mathrm{La}_{2} \mathrm{O}_{3}-$ $\mathrm{MgO}$ doped $\mathrm{Si}_{3} \mathrm{~N}_{4}$ ceramics.

The reconstructed electrostatic and absorptive potentials across the IGF are presented in Figs. 5(b) and 5(c) respectively. Figure $5(\mathrm{~b})$ shows that the IGF also has a less negative electrostatic potential than the $\mathrm{Si}_{3} \mathrm{~N}_{4}$ grains adjacent to it. The slanted region $\mathrm{AA}^{\prime}$ of the profile just beside grain 1 has no equivalent in the absorptive potential profile. The measured potential well width is $2.2 \mathrm{~nm}$ which agrees well, within the resolution limit of the present method, with the FWHM of the electrostatic potential profile of an IGF of the same material $(2.03 \mathrm{~nm})$ as investigated by Koch et al. Just as in the previous example, in $\mathrm{Lu}_{2} \mathrm{O}_{3}-\mathrm{MgO}$ doped $\mathrm{Si}_{3} \mathrm{~N}_{4}$ ceramics, $\mathrm{Lu}$ atoms segregate at the IGF/grain interface when a prism plane of $\beta$ $\mathrm{Si}_{3} \mathrm{~N}_{4}$ constitutes the interface plane. ${ }^{13), 14)}$ Here again grain 1 is a grain with high aspect ratio bordering the IGF with one of its long sides (the prism plane).

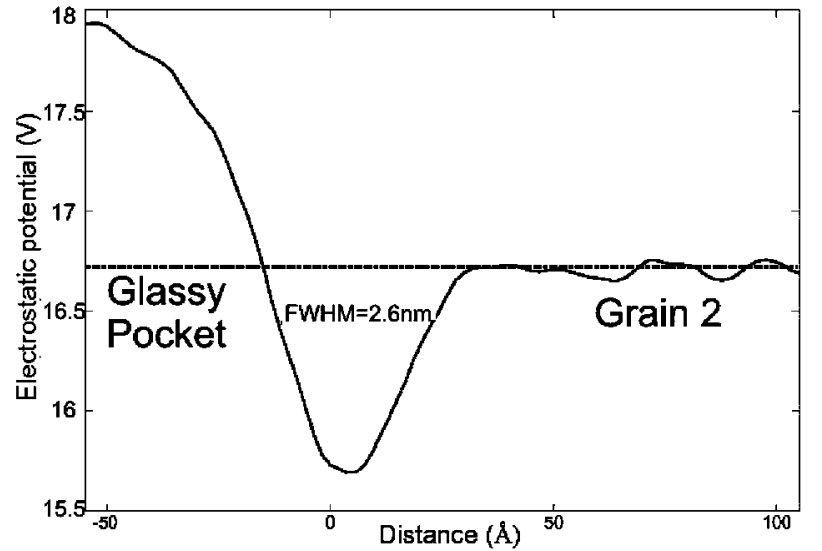

(a)

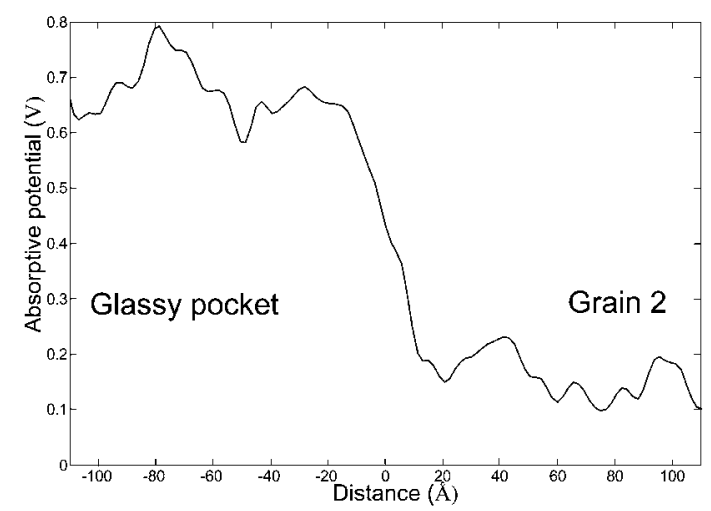

(b)

Fig. 4. (a) Reconstructed electrostatic potential (the potential was assumed to be constant for $15.8 \AA<x<105.2 \AA$ ) and (b) reconstructed absorptive potential of IGF/glassy interface.

\section{Discussion}

Comparing the absorptive potentials of the La- and Lu-containing ceramics (Figs. $3(\mathrm{~d})$ and $5(\mathrm{c})$ ) the Lu-containing IGF $\left(Z_{\mathrm{Lu}}=71\right)$ has an approximately $40 \%$ larger inelastic and high-angle scattering 'pseudo-absorptive' potential than the La-containing one. This is to be expected from the much higher nuclear charge of $\mathrm{Lu}$, even if $\mathrm{Lu}$ - and La-concentrations within the amorphous film are almost equal.

Generally, one would expect the absorptive and the mean inner potential to be approximately proportional. However, since the pseudo-absorptive potential also includes the highangle scattering, this proportionality constant depends very much on the size of the objective aperture as well as the local composition and diffracting conditions. The potential profile across the grain/glassy pocket interface is therefore needed to separate the contribution of local chemistry and space charge to the electrostatic potential profile. Under the assumption that space charge vanishes at large distance from the interface, the electrostatic potential within the glassy pocket in Fig. 4(a) may be assigned entirely to the composition $\left(\mathrm{SiO}_{2}+\mathrm{MgO}+\right.$ $\left.\mathrm{La}_{2} \mathrm{O}_{3}\right)$ of the amorphous material. This rise in mean inner potential of the amorphous phase with respect to the $\mathrm{Si}_{3} \mathrm{~N}_{4}$ grains may be attributed to the presence of the (heavy) La atoms. Since La has been observed to segregate to the grain/ glass interface by HAADF-STEM, ${ }^{12)-14)}$ we don't expect the mean inner potential to drop below that of $\mathrm{Si}_{3} \mathrm{~N}_{4}$ anywhere. This is also confirmed by the (space-charge insensitive) absorptive potential shown in Fig. 4(b). Without knowing the 


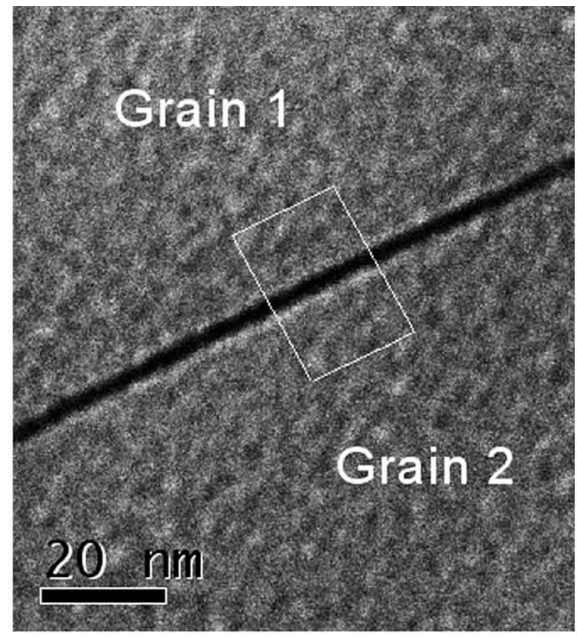

(a)

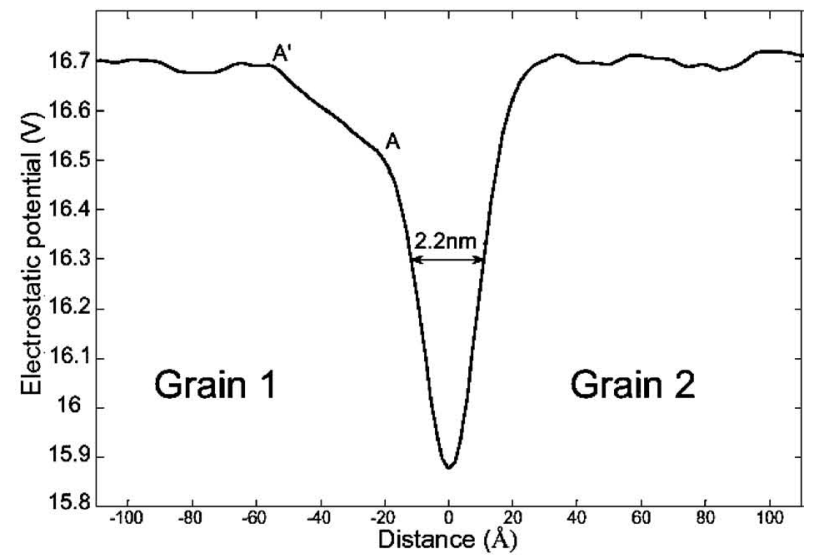

(b)

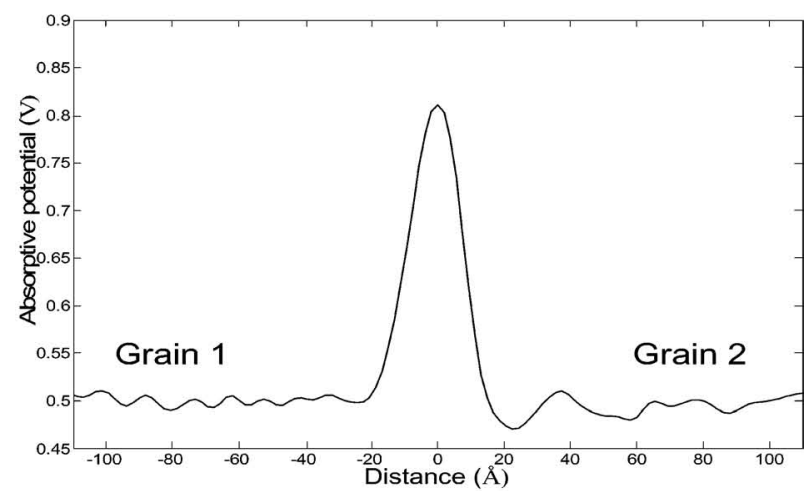

(c)

Fig. 5. (a) Image of an IGF in a $\mathrm{Lu}_{2} \mathrm{O}_{3}-\mathrm{MgO}$ doped $\mathrm{Si}_{3} \mathrm{~N}_{4}$ ceramic at an overfocus of $2 \mu \mathrm{m}$. (b) reconstructed electrostatic potential (the potential was assumed to be constant for $-137.8 \AA<x<-56.8 \AA$ and $32.6 \AA<x<133.2 \AA$ ) (c) reconstructed absorptive potential.

proportionality constant linking the electrostatic pseudoabsorptive potential magnitudes we may conclude that the mean inner potential of the amorphous material within the IGF and at the pocket/grain interface should be at least $1 \mathrm{~V}$ above that of the $\mathrm{Si}_{3} \mathrm{~N}_{4}$ grains. The observation of a drop of the electrostatic potential at these interfaces by more than $1 \mathrm{~V}$ may therefore only be explained by the presence of a space charge layer which is similar to the observations of Elfwing and Olsson ${ }^{16)}$ on the grain boundary of $\mathrm{ZnO}$ varistor material. A possible explanation is that the segregation of rare earth ions to the interface attracts compensating charge. This knowledge of space charge accumulation at IGFs and glassy pockets of $\mathrm{Si}_{3} \mathrm{~N}_{4}$ will help to validate MD and first principle simulation methods ${ }^{31)}$ for this material.

The slanted region of electrostatic potential profile indicated by $\mathrm{A}-\mathrm{A}^{\prime}$ in Fig. 5(b) may be interpreted as incomplete space charge screening within the IGF. However, further investigation of this phenomenon and additional experiments, such as spatially resolved spectroscopy are necessary for a more definite explanation to be found.

\section{Conclusions}

The projected electrostatic as well as the absorptive potential profiles across IGFs and grain/glassy pocket interface of $\mathrm{Si}_{3} \mathrm{~N}_{4}$ ceramics were obtained from the reconstructed exit face wave function (using POA). Since the electrostatic as well as the absorptive potentials were scaled with respect to vacuum, an absolute scale could be assigned to them. As the estimates for the electrostatic potentials are absolute, this method may replace off-axis electron holography, having the advantage that interfaces far from the specimen edge may be observed. A new measurement of IGF width, referred as 'potential well width', is proposed which is basically the FWHM of the potential well. For $\mathrm{La}_{2} \mathrm{O}_{3}-\mathrm{MgO}$ doped $\mathrm{Si}_{3} \mathrm{~N}_{4}$, the potential profile across the grain / glassy pocket interface was observed to be very similar to that across the IGF.

Acknowledgements The authors are thankful to Dr. Raphaelle Satet and Prof. Michael Hoffmann (University of Karlsruhe) for providing the samples used in the work and Dr. Anandh Subramanam for his help during experiments. Heartfelt thanks are expressed for Kersten Hahn, Mariya Sycha, Ute Bader and Ute Salzberger for their consistent support. Financial assistance from the European Commission under contract Nr. G5RD-CT2001-00586 (NANOAM) is acknowledged.

\section{References}

1) Kleebe, H.-J., J. Ceram. Soc. Japan, Vol. 105, pp. 453-475 (1997).

2) Kleebe, H.-J., J. Eur. Ceram. Soc., Vol. 10, pp. 151-159 (1992).

3) Pan, X., Gu, H., Stemmer, S. and Rühle, M., Mater. Sci. Forum, Vol. 207-209, pp. 421-424 (1996).

4) Brydson, R., Chen, S.-C., Riley, F. L., Milne, S. J., Pan, X. and Rühle, M., J. Am. Ceram. Soc., Vol. 81[2], pp. 369-379 (1998).

5) Jeong, H. G., Hiraga, K., Mabuchi, M. and Higashi, K., Philos. Mag. Lett., Vol. 74, pp. 73-80 (1996).

6) Avishai, A., Scheu, C. and Kaplan, W. D., Z. Metallkd., Vol. 94, pp. 272-276 (2003).

7) Chiang, Y. M., Silverman, L. A., French, R. F. and Cannon, R. M., J. Am. Ceram. Soc., Vol. 77, pp. 1143-1152 (1994).

8) Daschowdhury, K., Carpenter, R. W. and Braue, W., Ultramicroscopy, Vol. 40, pp. 229-239 (1992).

9) Clarke, D. R., Ultramicroscopy, Vol. 4, pp. 33-44 (1979).

10) Williams, D. B. and Romig, A. D., Ultramicroscopy, Vol. 30, pp. 38-51 (1989).

11) Gu, H., Pan, X., Cannon, R. M. and Rühle, M., J. Am. Ceram. Soc., Vol 81, pp. 3125-3135 (1998).

12) Shibata, N., Pennycook, S. J., Gosnell, T. R., Painter, G. S., Shelton W. L. and Becher, P. A., Nature, Vol. 428, pp. 730-732 (2004).

13) Ziegler, A., Idrobo, J. C., Cinibulk, M. K., Kisielowski, C., Browning, N. D. and Ritchie, R. O., Science, Vol. 306, pp. 1768-1770 (2004).

14) Winkelman, G. B., Dwyer, C., Hudson, T. S., Nguyen-Manh, D., Döblinger, M., Satet, R. L., Hoffmann, M. J. and Cockayne, D. J. H., Philos. Mag. Lett., Vol. 84, pp. 755-762 (2004). 
15) Ziegler, A., Kisielowski, C., Hoffmann, M. J. and Ritchie, R. O., J. Am. Ceram. Soc., Vol. 86, pp. 1777-1785 (2003).

16) Elfwing, M. and Olsson, E., J. Appl. Phys., Vol. 92, pp. 5272-5281 (2002).

17) Koch, C. T., Bhattacharyya, S., Rühle, M., Satet, R. L. and Hoffmann, M., Microsc. microanal., Vol. 12[02], pp. 156-159 (2006).

18) Dunin-Borkowski, R. E., Ultramicroscopy, Vol. 83, pp. 193$216(2000)$.

19) Gabor, D., Rev. Mod. Phys., Vol. 28, pp. 260-260 (1956).

20) Bhattacharyya, S., Koch, C. T. and Rühle, M., Ultramicroscopy, Vol. 106[6], pp. 528-538 (2006).

21) Allen, L. J., McBride, W., O’Leary, N. L. and Oxley, M. P., Ultramicroscopy, Vol. 100, pp. 91-104 (2004).

22) Teague, M. R., J. Opt. Soc. Am., Vol. 73, pp. 1434-1441 (1983).

23) Ishizuka, K. and Allman, B., Microscopy Today, Vol. 13, pp. 22-24 (2005).

24) Satet, R. L. and Hoffmann, M. J., Key Engineering Materials,
Vol. 264-268, pp. 775-780 (2004).

25) Stobbs, W. M. and Ross, F. M., Evaluation of Advanced Semiconductor materials by Electron Microscopy, NATO ASI series B, D. Cherns (Ed.), Plenum Press, London, Vol. 203, pp. 183-202 (1989).

26) Bhattacharyya, S., Subramaniam, A., Koch, C. T. and Rühle, M., Mater. Sci. Eng. A, Vol. 422, pp. 92-101 (2006).

27) Egerton, R. F., Electron Energy-Loss Spectroscopy in the Electron Microscope, 2nd ed., Plenum Press, New York (1996) pp. 302-305.

28) Wang, Y. G. and Dravid, V. P., Philos. Mag. Lett., Vol. 82[8], pp. 425-432 (2002).

29) Spence, J. C. H., "Experimental High Resolution Electron Microscopy," Clarendon Press, Oxford (1981) pp. 93-119.

30) Petzow, G. and Herrmann, M., Structure and Bonding, Vol. 102, pp. 47-165 (2002).

31) Rulis, P., Chen, J., Ouyang, L., Ching, W. Y., Su, X. and Garofalini, S. H., Phys. Rev. B, Vol. 71, pp. 235317 (2005). 